\title{
Ein seltener Fall von Lipidpneumonie durch Amiodaron
}

\section{llektra Voulgareli Alexandra Chronaiou Dionisios Tsoukalas George Tsoukalas}

Department of Pneumology, Sotiria Hospital, Athen, Griechenland

Schlüsselwörter

Endogene Lipidpneumonie · Amiodaron .

Bronchoalveoläre Lavage · Prednisolontherapie

\section{Zusammenfassung}

Wir berichten über einen Fall endogener Lipidpneumonie infolge Langzeitanwendung ( $\geq 30$ Jahre) von Amiodaron zur Behandlung von Vorhofflimmern bei einer 76-jährigen Patientin kaukasischer Abstammung, die sich mit Husten und Dyspnoe bei uns vorstellte. Die endogene Lipidpneumonie ist eine seltene und unterdiagnostizierte Krankheit, die häufiger bei Erwachsenen auftritt. Sie verläuft normalerweise asymptomatisch und wird meist bei Patienten diagnostiziert, die klinisch instabil geworden sind, oder wenn im Thoraxröntgenbild eine abnorme Verschattung der Lunge erkennbar ist. In dem hier vorgestellten Fall erfolgte die Diagnosestellung

durch fiberoptische Bronchoskopie mit Gewinnung von Flüssigkeit nach bronchoalveolärer Lavage (BALF), in der fettbeladene Makrophagen (Oil-Red-O-Färbung) zu finden waren. Da die Anwendung ölhaltiger Produkte anamnestisch ausgeschlossen worden war, wurde Amiodaron für die wahrscheinlichste Ursache der Lipidpneumonie gehalten. Die Behandlung wurde von Amiodaron auf Digoxin umgestellt und die Patientin erhielt oral Prednisolon. Während der 2-jährigen Nachbeobachtungsdauer blieb die Patientin klinisch stabil und die radiologischen Zeichen gingen zurück.

(c) 2019 Die Autoren

\section{Einführung}

Die endogene Lipidpneumonie ist eine seltene Krankheit, die häufiger Erwachsene betrifft, jedoch meist unterdiagnostiziert ist. Sie kann mit einer Bronchialobstruktion [1], Systemerkrankungen wie rheumatoider Arthritis, Hodgkin-Lymphom und Wegener-Granulomatose assoziiert sein oder idiopathisch auftreten [2]. In der vorliegenden Arbeit berichten wir über den Fall einer 76-jährigen Frau, bei der die Diagnose einer endogenen Lipidpneumonie infolge Langzeitanwendung (30 Jahre) von Amiodaron gestellt wurde und die durch Absetzen von Amiodaron und Verabreichung von Prednisolon erfolgreich behandelt wurde. Amiodaron wurde dabei durch Digoxin ersetzt. Die meisten berichteten Fälle von Lipidpneumonie, die als exogen bedingt diag- nostiziert werden, sind Folge einer Aspiration oder Inhalation von Mineralölen oder pflanzlichen Ölen [3, 11]. Die Lipidpneumonie tritt überwiegend bei älteren Menschen sowie bei Kindern mit Entwicklungsstörungen auf, vermutlich weil diese Gruppen eher zur Aspiration neigen [15].

\section{Fallbericht}

Eine 76-jährige Frau kaukasischer Abstammung stellte sich wegen seit 4 Wochen bestehenden Hustens mit mukopurulentem Sputum und Dyspnoe in unserer pulmonologischen Ambulanz vor. Die Patientin war Nichtraucherin und hatte einige Jahre in einer Baumwollfabrik gearbeitet. Den anamnestischen Angaben zufolge hatte kein Kontakt mit Substanzen bestanden, die be-

\section{KARGER}

Fax +497614520714

information@karger.com

www.karger.com

\section{() 2019 S. Karger GmbH, Freiburg}

Accessible online at

www.karger.com/kkp
Dr. Ilektra Voulgareli

Department of Pneumology

Sotiria Hospital

Mesogion 152, 27 Athen 115 27, Griechenland

ilektravoul@gmail.com 
Tab. 1. Ausgewählte Ergebnisse der Lungenfunktionsprüfung

\begin{tabular}{llll}
\hline & beobachtet & vorhergesagt & vorhergesagt (\%) \\
\hline Forcierte Vitalkapazität (FVC) & 2,32 I & 2,59 I & 90 \\
Forciertes exspiratorisches & 1,27 I & 1,97 I & 65 \\
Volumen in 1 S (FEV ${ }_{1}$ ) & 73 & 76 & 96 \\
FEV $_{1} /$ FVC (\%) & 1,49 I & 2,04 I & 73 \\
Inspiratorische Kapazität & 4,22 I & 4,75 I & 89 \\
Totale Lungenkapazität & 1,97 I & 2,13 I & 92 \\
Residualvolumen & 10,71 & 19,48 & 55 \\
D Lco bereinigt für Hämoglobin & & & \\
\hline
\end{tabular}

Lungenfunktionstests, die eine mäßige restriktive Lungenerkrankung und eine deutlich eingeschränkte Diffusionskapazität zeigen. Unter inhalativen Bronchodilatatoren kam es nicht zu einer Besserung (Daten nicht dargestellt). D D

kanntermaßen mit einer Lipidpneumonie assoziiert sind. Sie war in der jüngeren Vergangenheit nicht verreist und hatte keine Haustiere. Wegen eines bestehenden Vorhofflimmerns nahm sie seit 30 Jahren Amiodaron (200 mg 1-mal täglich) und wurde darüber hinaus wegen Hypertonie, Diabetes mellitus Typ II und Hypothyroidismus behandelt. Die körperliche Untersuchung ergab einen guten Körper- und Ernährungszustand sowie eine Pulsfrequenz von 69/Minute und Blutdruckwerte von 110/70 mmHg. Die anderen Vitalzeichen lagen im Normbereich bei einer Sauerstoffsättigung $\left(\mathrm{S}_{\mathrm{p}} \mathrm{O}_{2}\right)$ der Raumluft von 95\%. Des Weiteren war bei der körperlichen Untersuchung der Patientin über beiden basalen Lungenabschnitten ein Knisterrasseln zu hören; Blässe, Ikterus, Zyanose, Trommelschlegelfinger oder eine Lymphadenopathie bestanden nicht. Die Blutwerte lagen im Normbereich. In der Lungenfunktionsprüfung zeigten sich eine mäßige restriktive Lungenerkrankung sowie eine verminderte Diffusionskapazität (Tab. 1).

$\mathrm{Da}$ in der Röntgenaufnahme bilaterale Infiltrate erkennbar waren, erfolgte eine hochauflösende Computertomografie (high-resolution computed tomography, HRCT). Diese zeigte Bereiche mit Milchglastrübungen insbesondere in den Unterlappen beider Lungenflügel sowie Konsolidierungen des terminalen Luftraums (Abb. 1a, b). 2 Jahre später waren die Verschattungen zurückgegangen (Abb. 1c).

Mögliche Differenzialdiagnosen, einschließlich atypische Pneumonie, interstitielle Lungenerkrankung und Tuberkulose, wurden radiologisch ausgeschlossen. Bei der Patientin wurde eine fiberoptische Bronchoskopie mit Bronchialspülung durchgeführt, bei der auch Flüssigkeit nach bronchoalveolärer Lavage (BALF) für immunologische Untersuchungen gewonnen wurde. Die Spülflüssigkeiten wurden zur Anfertigung eines Ausstrichs und Untersuchung auf säurefeste Bazillen sowie zur zytologischen Untersuchung eingeschickt. In der bakteriologischen Untersuchung wurden keine Mikroorganismen gefunden und es waren keine malignen Zellen nachweisbar.

Die Gesamtzellzahl in der BALF betrug 287 500/ml und umfasste Makrophagen (64\%), Lymphozyten (31\%), Neutrophile (3\%) und Eosinophile (2\%). Bei der Beurteilung der BALF mittels spezifischer Färbung und Kolorierung waren fettbeladene Makro- phagen erkennbar (Oil-Red-O-Färbung) (Abb. 2a, b). Die zytologische Untersuchung des Sputums zeigte extrazellulär gelegene Öltröpfchen. Diese Ergebnisse sprachen für eine Lipidpneumonie.

Die Patientin hatte nie Mineralöle oder pflanzlichen Öle aspiriert oder inhaliert und nie intra- oder extranasal paraffinhaltige Mittel zum Abschwellen verwendet. Nach Angaben in der Literatur kann die Langzeitanwendung von Amiodaron jedoch eine endogene Lipidpneumonie (Phospholipidose) verursachen [17]. Amiodaron ist eine kationische amphiphile Verbindung, die die intrazelluläre transmembranöse Phospholipidbewegung stört und durch ihren starken inhibitorischen Effekt auf die lysosomale Phospholipase 2 den Phospholipidabbau hemmt. Die medikamenteninduzierte Phospholipidose manifestiert sich in Form einer Schaumzellreaktion.

Da die Patientin in unserem Fall 30 Jahre lang Amiodaron (200 mg 1-mal täglich) eingenommen hatte, wurde dies für die wahrscheinlichste Ursache gehalten. Daher wurde Amiodaron abgesetzt und die Patientin erhielt stattdessen Digoxin (0,25 mg 1-mal täglich) zur Behandlung des Vorhofflimmerns. Außerdem wurde eine Behandlung mit Prednisolon (20 mg 1-mal täglich) oral eingeleitet, die über einen Zeitraum von 6 Monaten allmählich ausgeschlichen wurde.

\section{Diskussion}

Die Lipidpneumonie wird in eine endogene und eine exogene Form eingeteilt $[2,5,6]$. Die endogene Lipidpneumonie tritt im Vergleich zur exogenen Lipidpneumonie seltener auf und hat eine unklare Ätiologie. Sie kann mit metabolischen oder sekretorischen Anomalien des Cholesterins im alveolären Epithelgewebe sowie mit einer übermäßigen Freisetzung von Lipiden einhergehen, die von Histiozyten aufgenommen werden [2]. Man nimmt an, dass es sich bei der endogenen Lipidpneumonie um eine chronische Fremdkörperreaktion auf Fett handelt, die durch lipidbeladene Makrophagen [5] gekennzeichnet ist und meist mit einer Bronchialobstruktion einhergeht [1]. Ferner kann die endogene Lipidpneumonie mit Systemerkrankungen wie rheumatoider Arthritis, Hodgkin-Lymphom und Wegener-Granulomatose asso- 

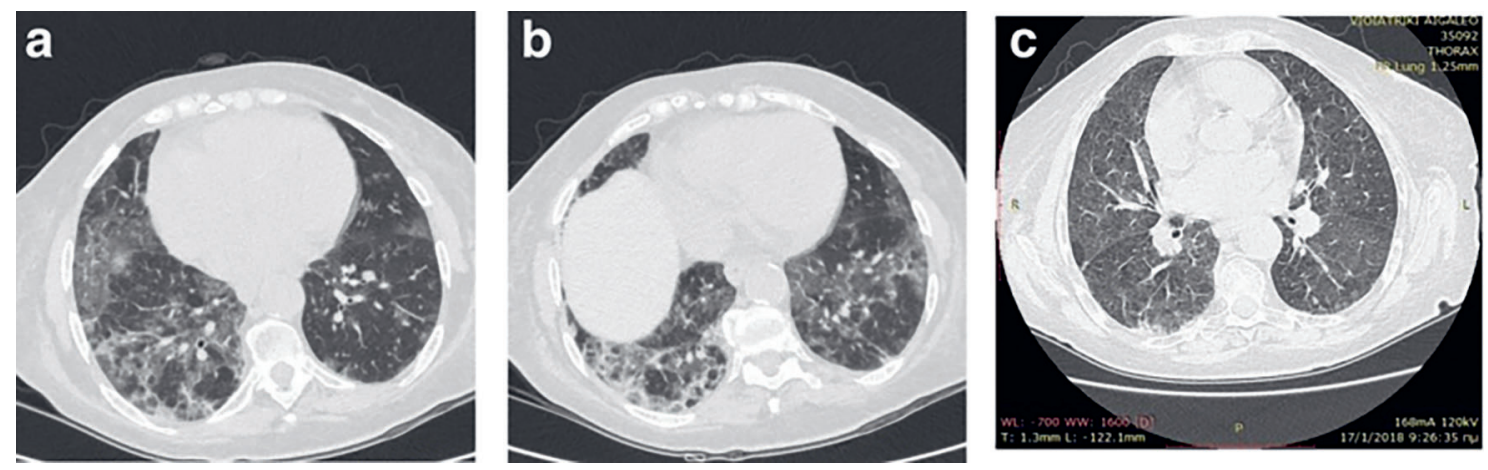

Abb. 1. Die Computertomografie-Aufnahmen einer 76-jährigen Frau mit Lipidpneumonie zeigten Milchglastrübungen und Bronchiektasien in beiden Lungenflügeln (a, b). 2 Jahre später waren die Verschattungen zurückgegangen (c).
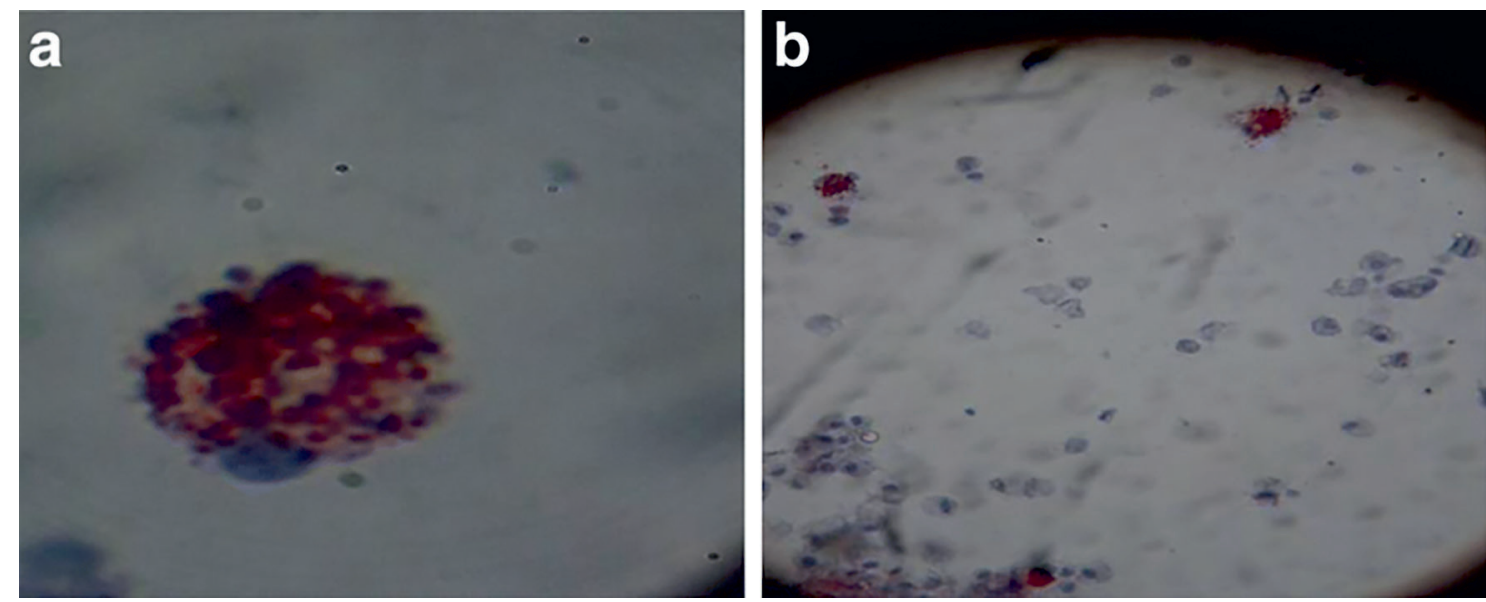

Abb. 2. (a, b) Untersuchung der BALF einer 76-jährigen Frau mit Lipidpneumonie. In der Oil-Red-O-Färbung war in der mikroskopischen Untersuchung die Phagozytose der Öltröpfchen durch alveoläre Makrophagen erkennbar.

ziiert sein. Darüber hinaus kann sie idiopathisch auftreten und ihre Pathogenese ist unbekannt [2]. Im vorliegenden Fall hatte die Patientin in der Vergangenheit keine ölhaltigen Mittel verwendet, weshalb die Diagnose einer endogenen Lipidpneumonie wahrscheinlicher war, zumal keine Systemerkrankung oder obstruktiven Veränderungen in der bildgebenden Diagnostik vorlagen. Da die Langzeitanwendung von Amiodaron nach Angaben in der Literatur eine endogene Lipidpneumonie verursachen kann, galt Amiodaron als verantwortliche Ursache für die Erkrankung der Patientin [15].

Amiodaron und seine Metaboliten können die Lunge durch einen zytotoxischen Effekt direkt schädigen und indirekt, indem sie eine immunologische Reaktion auslösen. Amiodaron kann die Bildung toxischer Sauerstoffradikale induzieren, die eine direkte Zellschädigung hervorrufen können. Zudem scheint Amiodaron die Akkumulation von Phospholipiden im Gewebe zu fördern. Es kommt zu einer Hyperplasie der Typ-II-Pneumozyten und einer Verbreiterung der Alveolarsepten mit einem zellulären entzündlichen Infiltrat und unterschiedlich stark ausgeprägter interstitieller Fibrose [18]. Bei der lichtmikroskopischen Untersuchung zeigte sich eine Vakuolisierung des Zytoplasmas in den alveolären Pneumozyten, Bronchialepithelzellen und Endothelzellen [16].
Die charakteristischen Merkmale und die Diagnose der Lipidpneumonie basieren auf dem Vorliegen lipidbeladener Makrophagen (den sogenannten Schaumzellen) in Atemwegsproben, wie Sputum, BALF oder mittels Feinnadelaspiration (FNA) gewonnenes Material zur zytologischen Untersuchung bzw. Biopsien aus Lungenläsionen, und natürlich auf einem hohen Verdachtsindex [2, 4, 7-9].

Typischerweise beginnt die Erkrankung schleichend mit unspezifischen Atemwegssymptomen wie Dyspnoe und/oder Husten. Eine akute Manifestation in Form einer akuten Atemwegserkrankung $[6,7,9,10]$ oder sogar mit Hämoptysen ist selten [13].

Die radiologischen Befunde können unterschiedlich ausfallen und einem Karzinom, einer akuten oder chronischen Pneumonie, einem akuten Atemnotsyndrom (ARDS) oder einem lokalisierten Granulom ähneln. Am besten eignet sich die HRCT für die Diagnose der Lipidpneumonie. Allerdings können die radiologischen Zeichen der Krankheit von denen einer Pneumonie, Lungenkrebs und/oder interstitiellen Lungenerkrankungen nicht zu unterscheiden sein [12]. In der Literatur wurde zudem berichtet, dass die Computer- und Magnetresonanztomografie in der Lage sind, Fett im Lungengewebe nachzuweisen. Zu den am häufigsten beschriebenen Merkmalen gehören alveoläre Konsolidierungen von 
geringer Abschwächung, Milchglastrübungen mit Verdickung der intralobulären Septen («Crazy-Paving»-Muster) oder alveoläre Knötchen [2, 6-8, 11].

Die Therapie der Lipidpneumonie ist nicht gut untersucht und die Erfahrungen sind auf Einzelfallberichte begrenzt. Einige Autoren haben die Anwendung systemischer Kortikosteroide vorgeschlagen, um die Entzündungsreaktion zu verlangsamen.

Kortikosteroide können jedoch nicht routinemäßig und nur bei schwerer und fortschreitender Lungenschädigung eingesetzt werden. Einige Autoren haben eine Resektion der am stärksten betroffenen Lungensegmente beschrieben, während andere Immunglobine verwenden oder eine Lungenspülung durchführen [2, 4, 7-9]. Unsere Patientin sprach gut auf orale Kortikosteroide an und ihr klinischer Zustand besserte sich im Verlauf der 2-jährigen Nachbeobachtung.

\section{Schlussfolgerung}

Insgesamt lässt sich sagen, dass es sich bei der endogenen Lipidpneumonie um eine seltene Erkrankung handelt, und in unserem Fall wurde die Diagnose auf die Langzeitanwendung von Amiodaron zurückgeführt.

Die Diagnose Lipidpneumonie wurde durch Untersuchung der BALF bestätigt, bei der sich schaumige Makrophagen zeigten, die in der Oil-Red-O-Färbung positiv waren [14]. Die tragende Säule unserer Behandlung bestand im Absetzen des verdächtigen Wirkstoffs und in einem Therapieversuch mit oralen Kortikosteroiden. Bleibt die Lipidpneumonie unentdeckt, kann sie schleichend oder symptomatisch verlaufen und zu einer Lungenfibrose und terminalen Lungenerkrankung führen.

\section{Danksagung}

Die Autoren danken Dr. Clio P. Mavragani (Department of Physiology, School of Medicine, University of Athens) für die hilfreichen Kommentare und Hinweise bei der Überprüfung des Manuskripts.

\section{Finanzielle Unterstützung}

Diese Forschungsarbeit erhielt keine finanzielle Unterstützung durch öffentliche Mittelgeber oder Finanzierungsagenturen aus dem kommerziellen oder Profit-Sektor.

\section{Verfügbarkeit der Daten und Materialien}

Die in dieser Arbeit verwendeten und/oder analysierten Datensätze sind auf Anfrage vom Korrespondenzautor erhältlich.

\section{Autorenbeiträge}

I.V. und A.C. bereiteten das Manuskript zur Einreichung vor. Vor der Einreichung wurde die Endversion des Manuskripts von allen Autoren genehmigt.

\section{Zustimmung zur Veröffentlichung}

Eine schriftliche Einverständniserklärung der Patientin für die Veröffentlichung des vorliegenden Fallberichts und der dazugehörigen Daten wurde eingeholt.

\section{Disclosure Statement}

Die Autoren erklären, dass keine Interessenskonflikte bestehen.

\section{Lizenzangabe}

Ilektra Voulgareli, Alexandra Chronaiou, Dionisios Tsoukalas, George Tsoukalas: A rare case of lipoid pneumonia attributed to amiodarone. Pneumonia 2018;10:12 (https://doi.org/10.1186/s41479-018-0056-3), ( The Author(s) 2018 (Übersetzung, «Publisher's note» gekürzt), lizensiert unter CC BY 4.0 (https://creativecommons.org/licenses/by/4.0/deed.de).

\section{Literatur}

1 Betancourt SL, Martinez-Jimenez S, Rossi SE et al.: Lipoid pneumonia: spectrum of clinical and radiologic manifestations. AJR Am J Roentgenol 2010;194:103-109.

-2 Lin J, Huang LL, Zhang JW, et al.: Endogenous lipoid pneumonia presenting as solitary pulmonary nodule: a case report. Int J Clin Exp Pathol 2015;8:9727-9730.

3 Kilaru H, Prasad S, Radha S, et al.: Nasal application of petrolatum ointment - a silent cause of exogenous lipoid pneumonia: successfully treated with prednisolone. Respir Med Case Rep 2017;22:98-100.

$\checkmark 4$ Annobil SH, Benjamin B, Kameswaran M, et al.: Lipoid pneumonia in children following aspiration of animal fat (ghee). Ann Trop Paediatr 1991;11:87-94.

5 Hadda V, Khilnani GC: Lipoid pneumonia: an overview. Expert Rev Respir Med 2010;4: 799-807.
6 Schwaiblmair M, Berghaus T, Haeckel T, et al.: Lipoid pneumonia - an underestimated syndrome. Dtsch Med Wochenschr 2010;135: 27-31.

7 Khilnani GC, Hadda V: Lipoid pneumonia: an uncommon entity. Indian J Med Sci 2009;63: 474-480.

8 Marchiori E, Zanetti G, Mano CM, et al.: Exogenous lipoid pneumonia. Clinical and radiological manifestations. Respir Med 2011;105: 659-666.

9 Hadda V, Khilnani GC, Bhalla AS, et al.: Lipoid pneumonia presenting as non-resolving community acquired pneumonia: a case report. Cases 2009;9332:J2.

10 Sharma A, Ohri S, Bambery P, et al.: Idiopathic endogenous lipoid pneumonia. Indian J Chest Dis Allied Sci 2006;48:143-145.

$>11$ Osman GA, Ricci A, Terzo F, et al.: Exogenous lipoid pneumonia induced by nasal decongestant. Clin Respir J 2016;12:524-531.

12 Wang YX, Fang F, Guo YF, et al.: Analysis of 12 cases of exogenous lipoid pneumonia confirmed by pathology. Zhonghua Jie $\mathrm{He} \mathrm{He} \mathrm{Hu}$ Xi Za Zhi 2017;40:445-449.
3 Simmons A, Rouf E, Whittle J: Not your typical pneumonia: a case of exogenous lipoid pneumonia. J Gen Intern Med 2007;22: 1613-1616.

14 Silverman JF, Turner RC, West RL, et al.: Bronchoalveolar lavage in the diagnosis of lipoid pneumonia. Diagn Cytopathol 1989;5:3-8.

15 Sachdev A, Anand P, Gupta D: Lipoid pneumonia - an unusual cause of acute respiratory distress syndrome. Indian Pediatr 2015;52: 63-64.

16 Wolkove N, Baltzan M: Amiodarone pulmonary toxicity. Can Respir J 2009;16:43-48.

17 Papiris SA, Triantafillidou C, Kolilekas L: Amiodarone: review of pulmonary effects and toxicity. Drug Saf 2010;33:539-558.

18 Miller BE, Hook GE: Hypertrophy and hyperplasia of alveolar type II cells in response to silica and other pulmonary toxicants. Environ Health Perspect 1990;85:15-23. 